NUB-3216

hep-th/0106244

\title{
Renormalization of Boundary Fermions AND World-Volume Potentials on D-BRAnes ${ }^{\star}$
}

\author{
P. Khorsand and T.R. Taylor \\ Department of Physics, Northeastern University, Boston, MA 02115, U.S.A.
}

\begin{abstract}
We consider a sigma model formulation of open string theory with boundary fermions carrying Chan-Paton charges at the string ends. This formalism is particularly suitable for studying world-volume potentials on D-branes. We perform explicit twoloop computations of the potential T-dual to the non-abelian Born-Infeld action. We also discuss the world-volume couplings of NS fluxes which are responsible for Myers' dielectric effect.
\end{abstract}

${ }^{\star}$ Research supported in part by the National Science Foundation under grant PHY-99-01057.

June 2001 


\section{Introduction}

Open strings end on D-branes. At low energies, their gauge degrees of freedom are described by a non-linear generalization of Maxwell's electrodynamics - the Born-Infeld theory on the D-brane world-volume. When a number $N$ of D-branes occupy the same hypersurface, the gauge group gets enhanced to $U(N)$. The world-volume action of $N$

coincident D-branes is described then by a non-abelian generalization of Born-Infeld theory (NBI). In spite of many efforts to unveil the structure of NBI action, its detailed form still remains elusive [1].

There are several methods available for studying D-brane actions. One of the most effective ones relies on the computations of scattering amplitudes for $U(N)$ gauge bosons. In fact, most results and conjectures are based on the higher-derivative corrections to fourpoint amplitudes analyzed by Tseytlin [2] in order to extract all interaction terms up to the fourth order in the gauge field strength $F$. Unfortunately, it is very difficult to push this type of computations any further since they involve amplitudes with larger numbers of external gauge bosons, a very difficult task indeed until a good recursive technique becomes available. Another method is based on world-sheet sigma model computations. Here, the world-volume action is reconstructed from the equations of motion which are obtained by requiring conformal invariance on the world-sheet. However, these computations [3, [4] appear to be quite complicated. There has not been much work done in this direction over the past few years.

The reason why the sigma model approach becomes difficult in the presence of nonabelian gauge symmetry is the non-linear (Wilson loop) coupling of the open string boundary $\partial \Sigma$ to the gauge field background. For the bosonic string, the relevant part of the sigma model action for string embeddings $X^{\mu}(z, \bar{z})$ coupled to the background gauge fields $A_{\mu}(X)$ is

$$
S=\frac{1}{2 \pi \alpha^{\prime}} \int_{\Sigma} d^{2} z \partial X^{\mu} \bar{\partial} X_{\mu}+S_{\partial \Sigma}(A) .
$$


The boundary coupling is given by the Wilson loop:

$$
e^{-S_{\partial \Sigma}(A)}=\operatorname{Tr} \mathcal{P} e^{i \oint_{\partial \Sigma} d \tau A_{\mu}(X) \partial_{\tau} X^{\mu}}
$$

where $A_{\mu}(X)$ are Hermitian matrices in the fundamental representation of the gauge group algebra and $\mathcal{P}$ is the path-ordering operation. Here, $\partial_{\tau}$ denotes the tangential derivative. In the traditional sigma model approach, one splits the embeddings into classical and quantum parts, expands the gauge field background $A_{\mu}(X)$ around the classical part and integrates over the quantum string fluctuations. The classical field is treated then as a sigma model coupling and the requirement that the corresponding beta function be zero is interpreted as its equation of motion. However, this procedure involves the Wilson loop expansion and leads to complicated Feynman diagrams.

In the case of D-branes of codimension $d>0$, the sigma model background includes also the scalar fields $\phi_{i}, i=1, \ldots, d$, in the adjoint representation of the gauge group. They describe brane oscillations in the directions transverse to the rigid hypersurface. These fields are associated with the world-sheet coordinates $X^{i}$ satisfying the Dirichlet boundary conditions, $X^{i}(\partial \Sigma)=0$, and their boundary coupling is

$$
e^{-S_{\partial \Sigma}(\phi)}=\operatorname{Tr} \mathcal{P} e^{i \oint_{\partial \Sigma} d \tau \phi_{i}(X) \partial_{r} X^{i}}
$$

where $\partial_{r}$ denotes the normal derivative. The main focus of our work is the effective potential for these non-abelian scalars. In fact, the scalar lagrangian is related to the gauge lagrangian by T-duality and can be used to extract the NBI action.

This paper is organized as follows. In Section 2, we reformulate the sigma model by introducing boundary fermions that carry Chan-Paton charges at the string ends. In Section 3, we describe the one-loop computation of anomalous dimensions of these fermions and of the beta function associated to non-abelian scalar couplings. We extend our discussion to two loops for bosonic strings and superstrings in Sections 4 and 5, respectively. In particular, we derive the world-volume D-brane potentials and discuss the relation of our 
results to previous sigma model computations of the NBI action. In Section 6, we study the effects of background NS fluxes up to two loops in the sigma model expansion. At one loop, we recover Myers' dielectric coupling [5] in both bosonic and in superstring cases. We also show that this coupling does not receive any two-loop corrections.

\section{Sigma model with boundary fermions}

It is well known that gauge degrees of freedom can be introduced in open string theory by attaching to the string boundary a set of complex Grassmann valued fields $\lambda_{a}$, $a=1, \ldots, N$, in the fundamental representation of $U(N)$. Quantization of these fermions generates $N$ conserved charges attached to the string boundary and generates Chan-Paton factors. In the context of sigma model, the Wilson loop action can be rewritten [6] as a functional integral over the boundary fermions coupled to the string coordinates $X^{i}$ via $N \times N$ Hermitian, traceless matrices $\phi_{i}$ :

$$
e^{-S_{\partial \Sigma}(\phi)}=\sum_{\theta} \int_{\mathrm{AP}}\left[d \lambda^{\dagger} d \lambda\right] e^{i \theta\left[\lambda^{\dagger} \lambda\left(\tau=\tau_{0}\right)+\frac{N}{2}-1\right]} e^{-\int_{0}^{2 \pi} d \tau\left(\lambda^{\dagger} \frac{d}{\partial \tau} \lambda-i \partial_{r} X^{i} \lambda^{\dagger} \phi_{i} \lambda\right)}
$$

The fermions satisfy antiperiodic (AP) boundary conditions on the interval $\tau \in[0,2 \pi]$. The summation, which extends over the angles $\theta=2 \pi k / N$ with $k=1, \ldots, N$, must be included in order to project the intermediate states in the path integral on coherent states of occupation number 1 . The point $\tau_{0}$ can be arbitrarily chosen on the boundary. In this work, we study the renormalization properties of the sigma model reformulated by using these boundary fermions.f We limit our considerations to the case of constant, spacetimeindependent backgrounds $\phi_{i}$.

We first consider quantum effects due to fluctuations of string embeddings $X^{i}$. Integration over these fluctuations leads to ultraviolet divergences. We focus on the loop

\footnotetext{
${ }^{1}$ The traces describe the center of mass motion which decouples from other degrees of freedom.

${ }^{2}$ See [7] for a partial list of references utilizing similar "auxiliary" fermions.
} 
corrections to the correlation functions that determine the beta function $\beta_{i}(\phi)$. Since the classical theory is conformally invariant, we are free to work on a disk, parameterized by $(r, \tau)$, with the boundary at $r=1$. The bosonic string fluctuations couple to the boundary through their derivatives $\partial_{r} X^{i}$, therefore the corresponding Feynman diagrams involve the two-point functions

$$
\left.\left\langle\partial_{r} X^{i}(\tau) \partial_{r^{\prime}} X^{j}\left(\tau^{\prime}\right)\right\rangle \equiv\left\langle\partial_{r} X^{i}(r, \tau) \partial_{r^{\prime}} X^{j}\left(r^{\prime}, \tau^{\prime}\right)\right\rangle\right|_{r=r^{\prime}=1}=\left.\delta^{i j} \frac{\partial}{\partial r} \frac{\partial}{\partial r^{\prime}} \mathcal{D}\left(r, \tau ; r^{\prime}, \tau^{\prime}\right)\right|_{r=r^{\prime}=1}
$$

where $\mathcal{D}$ is the Dirichlet propagator. The r.h.s. is related to the Neumann propagator $\mathcal{N}$ by a T-duality relation,

$$
\left.\frac{\partial}{\partial r} \frac{\partial}{\partial r^{\prime}} \mathcal{D}\left(r, \tau ; r^{\prime}, \tau^{\prime}\right)\right|_{r=r^{\prime}=1}=-\left.\frac{\partial}{\partial \tau} \frac{\partial}{\partial \tau^{\prime}} \mathcal{N}\left(r, \tau ; r^{\prime}, \tau^{\prime}\right)\right|_{r=r^{\prime}=1}=\frac{\alpha^{\prime}}{2 \sin ^{2}\left[\frac{\left(\tau-\tau^{\prime}\right)}{2}\right]}
$$

It is convenient to define

$$
D\left(\tau-\tau^{\prime}\right) \equiv \frac{\alpha^{\prime}}{2 \sin ^{2}\left[\frac{\left(\tau-\tau^{\prime}\right)}{2}\right]},
$$

so that

$$
\left\langle\partial_{r} X^{i}(\tau) \partial_{r^{\prime}} X^{j}\left(\tau^{\prime}\right)\right\rangle=D\left(\tau-\tau^{\prime}\right) \delta^{i j}
$$

The sigma model contains only one type of interaction,

$$
S_{I}=i \int_{0}^{2 \pi} d \tau \partial_{r} X^{i} \lambda_{a}^{\dagger}\left(\phi_{i}\right)^{a b} \lambda_{b}
$$

which is essentially a QED-like vertex with $d N \times N$ coupling matrices $\phi_{i}$.

Finally, the free AP fermionic propagator is

$$
\Delta_{a b}^{(0)}\left(\tau, \tau^{\prime}\right)=\left\langle\lambda_{a}(\tau) \lambda_{b}^{\dagger}\left(\tau^{\prime}\right)\right\rangle=\frac{i}{2} \delta_{a b} \operatorname{sign}\left(\tau-\tau^{\prime}\right)
$$




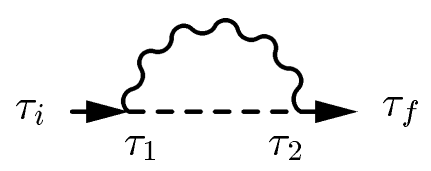

A

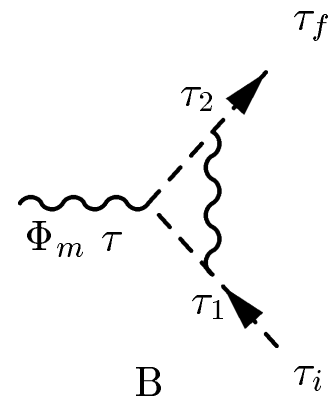

Figure 1: One-loop correction to the boundary fermion propagator (A) and to the boundary fermion-string vertex (B). Bosonic string coordinates are represented by wiggly lines while boundary fermions by dashed lines.

\section{One-loop computations}

Although the one loop computation is very simple, it is quite instructive to outline some details. We begin with the one-loop correction to the fermion propagator shown on Fig.1A:

$$
\begin{aligned}
& \frac{i}{2} \operatorname{sign}\left(\tau_{i}-\tau_{f}\right) \Delta_{a b}^{(1)}\left(\tau_{i}, \tau_{f}\right)= \\
& \quad \frac{i}{8}\left(\phi_{i} \phi_{i}\right)_{a b} \int_{0}^{2 \pi} d \tau_{1} \int_{0}^{2 \pi} d \tau_{2} \operatorname{sign}\left(\tau_{i}-\tau_{1}\right) D\left(\tau_{1}-\tau_{2}\right) \operatorname{sign}\left(\tau_{1}-\tau_{2}\right) \operatorname{sign}\left(\tau_{2}-\tau_{f}\right) .
\end{aligned}
$$

Note that the integration over the interaction points $\tau_{1}$ and $\tau_{2}$ extends a priori over the whole range of angles from 0 to $2 \pi$, inside and outside the interval $\left[\tau_{i}, \tau_{f}\right]$, with different integration regions contributing $\int d \tau_{1} \int d \tau_{2} D\left(\tau_{1}-\tau_{2}\right)$ with \pm signs depending on the relative position of the angles. We display the relevant signs on Fig.2A. We also show zeroes for regions that cancel as a consequence of $\tau_{1} \leftrightarrow \tau_{2}$ symmetry of the $D$-propagator. Since according to the relation (2.3), the $D$-propagator is a double derivative of a periodic function:

$$
D\left(\tau-\tau^{\prime}\right)=-\frac{\partial^{2} \mathcal{N}}{\partial \tau \partial \tau^{\prime}}, \quad \mathcal{N}\left(\tau-\tau^{\prime}\right)=-\alpha^{\prime} \ln \sin ^{2}\left[\frac{\left(\tau-\tau^{\prime}\right)}{2}\right]
$$

the sum of contributions in each row or column of Fig.2A is zero. This fact can be used to rearrange the integration regions as shown in Fig.2B. Finally, by using the $\tau_{1} \leftrightarrow \tau_{2}$ 


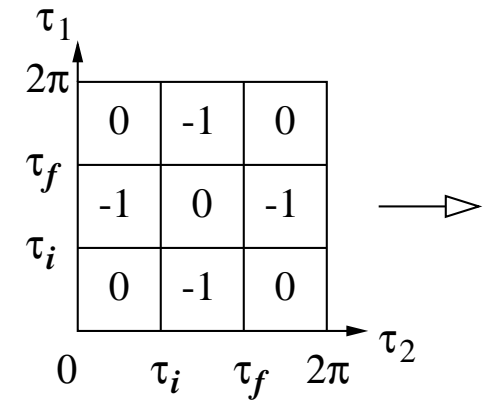

A

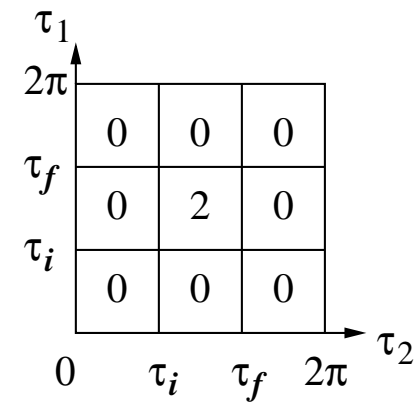

B

Figure 2: Weights of contributions to the one-loop propagator from all integration regions, before $(\mathrm{A})$ and after $(\mathrm{B})$ the rearrangement that leads to path-ordering.

symmetry one obtains

$$
\Delta_{a b}^{(1)}\left(\tau_{i}-\tau_{f}\right)=\left(\phi_{i} \phi_{i}\right)_{a b} \mathcal{P} \int_{\tau_{i}}^{\tau_{f}} d \tau_{1} d \tau_{2} D\left(\tau_{1}-\tau_{2}\right)
$$

so the vertices become path-ordered. As a result, the perturbation theory becomes pathordered, like in ordinary quantum mechanics. In the sigma model framework, this amounts to Chan-Paton rules for inserting the interaction vertex (2.6).

The integral (3.3) is divergent. In fact, it contains two types of singularities: linear divergence along the line $\tau_{1}=\tau_{2}$ and logarithmic "end-point" singularities. The linear divergence is spurious and can be avoided by a formal integration:

$$
\mathcal{P} \int_{\tau_{i}}^{\tau_{f}} d \tau_{1} d \tau_{2} D\left(\tau_{1}-\tau_{2}\right)=\frac{1}{2} \int_{\tau_{i}}^{\tau_{f}} d \tau_{1} \int_{\tau_{i}}^{\tau_{f}} d \tau_{2} D\left(\tau_{1}-\tau_{2}\right)=\mathcal{N}\left(\tau_{i}-\tau_{f}\right)-\mathcal{N}(0)
$$

The remaining logarithmic singularity $\mathcal{N}(0)$ can be regulated by introducing a shortdistance cutoff $\Lambda$. As a result, one obtains

$$
\Delta_{a b}^{(1)}\left(\tau_{i}-\tau_{f}\right)=\alpha^{\prime}\left(\phi_{i} \phi_{i}\right)_{a b} \ln \left(\frac{\Lambda}{\sin ^{2}\left[\frac{\left(\tau_{i}-\tau_{f}\right)}{2}\right]}\right)
$$

The corresponding one-loop anomalous dimension matrix of boundary fermions is

$$
\gamma^{(1)}=\alpha^{\prime} \phi_{i} \phi_{i}
$$


The one-loop correction to the interaction term (2.6) is shown on Fig.1B. For our purposes, it is sufficient to consider the one-particle irreducible three-point function $\Phi_{m}\left(\tau_{i}, \tau, \tau_{f}\right)$, with the tree-level coupling $\phi_{m}$ inserted at $\tau \in\left[\tau_{i}, \tau_{f}\right]$. By rearranging integrals in a manner similar to the fermion propagator, one can rewrite this diagram as a path-ordered integral, with the position of interaction points correlated with the order of non-abelian couplings in a way dictated by Chan-Paton rules:

$$
\Phi_{m}^{(1)}\left(\tau_{i}, \tau, \tau_{f}\right)=\phi_{i} \phi_{m} \phi_{i} \int_{\tau_{i}}^{\tau} d \tau_{1} \int_{\tau}^{\tau_{f}} d \tau_{2} D\left(\tau_{1}-\tau_{2}\right)
$$

The regularized integral yields

$$
\Phi_{m}^{(1)}\left(\tau_{i}, \tau, \tau_{f}\right)=\alpha^{\prime} \phi_{i} \phi_{m} \phi_{i} \ln \left(\frac{\sin ^{2}\left[\frac{\left(\tau-\tau_{i}\right)}{2}\right] \sin ^{2}\left[\frac{\left(\tau-\tau_{f}\right)}{2}\right]}{\Lambda \sin ^{2}\left[\frac{\left(\tau_{i}-\tau_{f}\right)}{2}\right]}\right) .
$$

After combining this result with Eq.(3.6), we find the one-loop beta function

$$
\beta_{m}^{(1)}=\alpha^{\prime}\left[\phi_{i},\left[\phi_{i}, \phi_{m}\right]\right]
$$

The requirement of vanishing one-loop beta function, i.e. the non-renormalization of $\phi$ couplings leads to equations of motion that can be obtained by varying the well-known D-brane potential:

$$
\frac{\delta}{\delta \phi_{m}} \frac{\alpha^{\prime}}{2} \operatorname{Tr}\left(\phi_{i j} \phi_{j i}\right)=\alpha^{\prime}\left[\phi_{i}, \phi_{i m}\right]=0
$$

where

$$
\phi_{i j} \equiv\left[\phi_{i}, \phi_{j}\right]
$$

\section{Two loops in bosonic string theory}

With the sigma model reformulated by using the boundary fermions, the analysis of higher loop orders becomes much simpler than in the traditional approach. All diagrams are QED-like, with path-ordered interaction vertices. In particular, the two-loop computation can be performed explicitly. There are two diagrams, shown on Fig.3, which contribute to the two-loop anomalous dimensions. They give, respectively, 


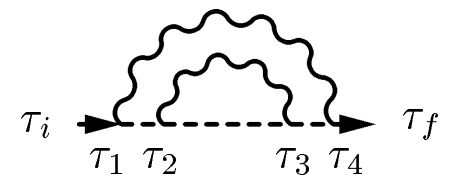

A

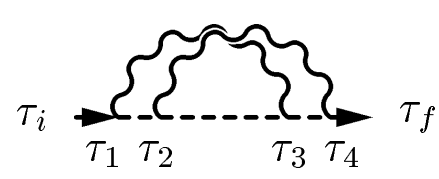

B

Figure 3: Two-loop corrections to the boundary fermion propagator.

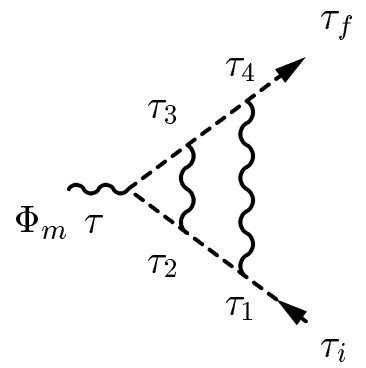

A

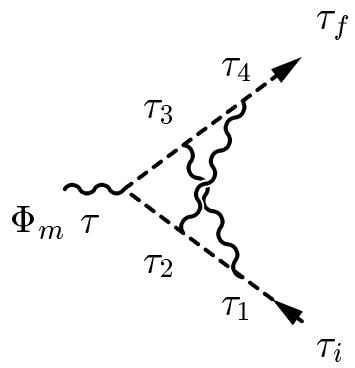

B

Figure 4: Two-loop corrections to the boundary fermion-string vertex.

$$
\begin{aligned}
& \Delta_{a b}^{(3 A)}=\left(\phi_{i} \phi_{j} \phi_{j} \phi_{i}\right)_{a b} \mathcal{P} \int_{\tau_{i}}^{\tau_{f}} d \tau_{1} d \tau_{2} d \tau_{3} d \tau_{4} D\left(\tau_{1}-\tau_{4}\right) D\left(\tau_{2}-\tau_{3}\right) \\
& \Delta_{a b}^{(3 B)}=\left(\phi_{i} \phi_{j} \phi_{i} \phi_{j}\right)_{a b} \mathcal{P} \int_{\tau_{i}}^{\tau_{f}} d \tau_{1} d \tau_{2} d \tau_{3} d \tau_{4} D\left(\tau_{1}-\tau_{3}\right) D\left(\tau_{2}-\tau_{4}\right) .
\end{aligned}
$$

The divergent parts of the above integrals can be extracted by elementary integrations:

$$
\begin{aligned}
\mathcal{P} \int_{\tau_{i}}^{\tau_{f}} d \tau_{1} d \tau_{2} d \tau_{3} d \tau_{4} D\left(\tau_{1}-\tau_{4}\right) D\left(\tau_{2}-\tau_{3}\right) & =\left(\alpha^{\prime}\right)^{2}\left(\frac{1}{2} \ln ^{2} \Lambda-2 \ln \Lambda\right)+\ldots \\
\mathcal{P} \int_{\tau_{i}}^{\tau_{f}} d \tau_{1} d \tau_{2} d \tau_{3} d \tau_{4} D\left(\tau_{1}-\tau_{3}\right) D\left(\tau_{2}-\tau_{4}\right) & =\left(\alpha^{\prime}\right)^{2}\left(-\ln ^{2} \Lambda+2 \ln \Lambda\right)+\ldots
\end{aligned}
$$

In the above expressions, we neglected finite terms as well as $\ln \Lambda$ terms with "kinematic" coefficients $\ln \sin ^{2}\left[\frac{\left(\tau_{i}-\tau_{f}\right)}{2}\right]$ which are cancelled by one-loop counterterms. The two-loop anomalous dimensions can be read out from the single $\ln \Lambda$ divergences which, together

\footnotetext{
3 As a consistency check, it is easy to verify that the coefficients of $\ln ^{2} \Lambda$ terms agree with the renormalization group equations.
} 
with Eq.(3.6), yield

$$
\gamma=\alpha^{\prime} \phi_{i} \phi_{i}+\left(\alpha^{\prime}\right)^{2} \phi_{i j} \phi_{i j}+O\left[\left(\alpha^{\prime}\right)^{3}\right]
$$

The two-loop beta function can be computed in a similar way. There are only two diagrams, shown on Fig.4, that contribute single logarithms $\ln \Lambda$ to the vertex function $\Phi_{m}\left(\tau_{i}, \tau, \tau_{f}\right)$. They yield, respectively,

$$
\begin{aligned}
\Phi_{m}^{(4 A)} & =\phi_{i} \phi_{j} \phi_{m} \phi_{j} \phi_{i} \mathcal{P} \int_{\tau_{i}}^{\tau} d \tau_{1} d \tau_{2} \int_{\tau}^{\tau_{f}} d \tau_{3} d \tau_{4} D\left(\tau_{1}-\tau_{4}\right) D\left(\tau_{2}-\tau_{3}\right) \\
\Phi_{m}^{(4 B)} & =\phi_{i} \phi_{j} \phi_{m} \phi_{i} \phi_{j} \mathcal{P} \int_{\tau_{i}}^{\tau} d \tau_{1} d \tau_{2} \int_{\tau}^{\tau_{f}} d \tau_{3} d \tau_{4} D\left(\tau_{1}-\tau_{3}\right) D\left(\tau_{2}-\tau_{4}\right)
\end{aligned}
$$

For completeness, we list below the results of integrations:

$$
\begin{aligned}
\mathcal{P} \int_{\tau_{i}}^{\tau} d \tau_{1} d \tau_{2} \int_{\tau}^{\tau_{f}} d \tau_{3} d \tau_{4} D\left(\tau_{1}-\tau_{4}\right) D\left(\tau_{2}-\tau_{3}\right) & =\left(\alpha^{\prime}\right)^{2}\left(\frac{1}{2} \ln ^{2} \Lambda+2 \ln \Lambda\right)+\ldots \\
\mathcal{P} \int_{\tau_{i}}^{\tau} d \tau_{1} d \tau_{2} \int_{\tau}^{\tau_{f}} d \tau_{3} d \tau_{4} D\left(\tau_{1}-\tau_{3}\right) D\left(\tau_{2}-\tau_{4}\right) & =-2\left(\alpha^{\prime}\right)^{2} \ln \Lambda+\ldots
\end{aligned}
$$

After combining these results with the two-loop anomalous dimension of Eq.(4.5), we obtain the following two-loop contribution to the beta function:

$$
\beta_{m}^{(2)}=\left(\alpha^{\prime}\right)^{2}\left[\phi_{i j},\left[\phi_{i j}, \phi_{m}\right]\right]
$$

Unlike in the one-loop case, the non-renormalization condition, $\beta_{m}^{(2)}=0$, is not integrable, i.e. it cannot be obtained by varying a gauge-invariant potential. However, by using Jacobi identity, Eq. (4.10) can be rewritten as:

$$
\beta_{m}^{(2)}=\left(\alpha^{\prime}\right)^{2}\left\{\frac{\delta}{\delta \phi_{m}} \frac{2}{3} \operatorname{Tr}\left(\phi_{i j} \phi_{j k} \phi_{k i}\right)+2\left[\phi_{i m},\left[\phi_{j}, \phi_{j i}\right]\right]\right\} .
$$

Since the second term involves the one-loop beta function $\beta_{i}^{(1)}$ of Eq.(3.9), it is of higher order $O\left[\left(\alpha^{\prime}\right)^{3}\right]$. In this way, we obtain the D-brane potentialf

$$
V(\phi)=\frac{\alpha^{\prime}}{2} \operatorname{Tr}\left(\phi_{i j} \phi_{j i}\right)+\frac{2\left(\alpha^{\prime}\right)^{2}}{3} \operatorname{Tr}\left(\phi_{i j} \phi_{j k} \phi_{k i}\right)+O\left[\left(\alpha^{\prime}\right)^{3}\right]
$$

\footnotetext{
${ }^{4}$ The overall normalization is not fixed by the sigma model - it requires introducing the brane tension factor $T_{p}$.
} 
Higher loop computations are more difficult, mostly due to integrals associated to multiple insertions of the interaction vertices. In particular, the cutoff regularization leads to complicated integrals and should be replaced by a more efficient procedure like dimensional regularization.

The scalar potential of Eq.(4.12) is related by T-duality to the action for the gauge fields. Indeed, by replacing $\phi_{i j} \leftrightarrow i F_{\mu \nu}$ and $\left[\phi_{k}, \phi_{i j}\right] \leftrightarrow D_{\rho} F_{\mu \nu}$, one obtains the gauge kinetic terms and the $F^{3}$ term [8]. By a similar transformation on Eq.(4.10) one recovers the two-loop beta function computed previously in [3, 4]. More recently, Myers [5] proposed a complete form of the scalar potential in superstring theory, to all orders in $\alpha^{\prime}$, by relating it via T-duality to the Tseytlin's form of NBI action [9]. If the approach developed in this work could be extended to higher loops, it would be useful not only for computing scalar potentials but also for understanding the structure of NBI action. In the following Sections, we discuss superstrings and examine some aspects of Myers' proposal.

\section{Superstrings}

The superstring sigma model action is

$$
S=\frac{1}{2 \pi \alpha^{\prime}} \int_{\Sigma} d^{2} z\left(\partial X^{i} \bar{\partial} X^{i}+\frac{\alpha^{\prime}}{2} \Psi^{i} \bar{\partial} \Psi^{i}+\frac{\alpha^{\prime}}{2} \bar{\Psi}^{i} \partial \bar{\Psi}^{i}\right)+S_{\partial \Sigma}(\phi)
$$

where the world-sheet $\Sigma$ is parameterized by $z=r e^{i \tau}$ and $\bar{z}=r e^{-i \tau}$. Here, $\Psi^{i}$ and $\bar{\Psi}^{i}$ are the world-sheet fermions. The supersymmetrized Wilson loop corresponds to the interaction term:

$$
S_{I}=\int_{0}^{2 \pi} d \tau\left[i \partial_{r} X^{i} \lambda_{a}^{\dagger}\left(\phi_{i}\right)^{a b} \lambda_{b}+\alpha^{\prime} \psi^{i} \psi^{j} \lambda_{a}^{\dagger}\left(\phi_{i j}\right)^{a b} \lambda_{b}\right]
$$

where $\psi^{i}$ is the restriction of $\Psi^{i}$ to the boundary, subject to the Dirichlet condition.

$$
\left.\sqrt{i z} \Psi^{i}\right|_{\partial \Sigma}=-\left.\sqrt{-i \bar{z}} \bar{\Psi}^{i}\right|_{\partial \Sigma}=i \psi^{i}(\tau)
$$

\footnotetext{
${ }^{5}$ The factors of $\sqrt{i z}$ are due to the $z \rightarrow \tau$ change of variables.
} 


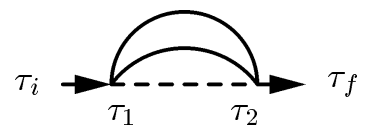

A

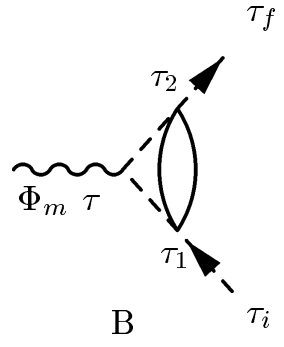

B

Figure 5: In superstring theory, starting at the two-loop level, world-sheet fermions give rise to additional contributions to the anomalous dimensions (A) and beta function (B). World-sheet fermions are represented by solid lines.

In the presence of two-form NS background field $B_{i j}(X)$ with non-vanishing field strength

$$
H_{i j k}=\partial_{i} B_{j k}+\partial_{k} B_{i j}+\partial_{j} B_{k i}
$$

the sigma model contains also the interaction terms which, up to the linear order in $B_{i j}(X)$, take the form:

$$
S_{B}=\frac{1}{2 \pi \alpha^{\prime}} \int_{\Sigma} d^{2} z\left[B_{i j}(X) \partial X^{i} \bar{\partial} X^{j}+i \frac{\alpha^{\prime}}{4} H_{i j k} \partial X^{i} \bar{\Psi}^{j} \bar{\Psi}^{k}-i \frac{\alpha^{\prime}}{4} H_{i j k} \bar{\partial} X^{i} \Psi^{j} \Psi^{k}\right] .
$$

In superstring theory, anomalous dimensions and beta functions receive additional contributions from the world-sheet fermions. Their propagators are [10], respectively,

$$
\left\langle\Psi^{i}(z) \Psi^{j}(w)\right\rangle=\frac{\delta^{i j}}{z-w}, \quad\left\langle\Psi^{i}(z) \bar{\Psi}^{j}(\bar{w})\right\rangle=\frac{i \delta^{i j}}{1-z \bar{w}} .
$$

It is also convenient to rewrite the boundary interaction term (5.2) as a complex contour integral:

$$
S_{I}=\oint_{\partial \Sigma} d u\left[\partial X^{i} \lambda_{a}^{\dagger}\left(\phi_{i}\right)^{a b} \lambda_{b}-\frac{\alpha^{\prime}}{2} \Psi^{i} \Psi^{j} \lambda_{a}^{\dagger}\left(\phi_{i j}\right)^{a b} \lambda_{b}\right]-c . c .
$$

When $B_{i j}=0$, the world-sheet fermions first contribute at the two-loop level. The relevant diagrams are shown on Fig.5. It is easy to see that the diagram 5A cancels the

\footnotetext{
${ }^{6}$ Note that as a result of analytic continuation to the Euclidean world-sheet this part of the action
} becomes purely imaginary. 
$O\left[\left(\alpha^{\prime}\right)^{2}\right]$ bosonic correction to the anomalous dimension (4.5) while the diagram 5B cancels the two-loop correction (4.10) to the beta function. As a result, the supersymmetric Dbrane potential does not receive $O\left[\left(\alpha^{\prime}\right)^{2}\right]$ corrections. This reflects the absence of $F^{3}$ terms in the supersymmetric NBI action.

\section{NS Fluxes}

As originally pointed out by Myers [5], and later confirmed by explicit computations of scattering amplitudes [1], the world-volume potentials involve also couplings of scalars to NS fluxes. ${ }^{7}$ We will first explain how these couplings emerge from the sigma model formalism.

Following the background field method, we introduce a harmonic background $\widetilde{X}^{i}$ and expand the interaction term (5.5):

$$
\widetilde{S}_{B}=\frac{1}{4 \pi \alpha^{\prime}} \int_{\Sigma} d^{2} z\left[\partial \widetilde{X}^{i} H_{i j k}\left(\bar{\partial} X^{j} X^{k}\right)+i \frac{\alpha^{\prime}}{2} \partial \widetilde{X}^{i} H_{i j k} \bar{\Psi}^{j} \bar{\Psi}^{k}\right]-\text { c.c. }+\ldots
$$

Quantum fluctuations can propagate from the interior of the disk to the boundary with the (Dirichlet) propagators [10

$$
\left\langle X^{i}(z) X^{j}(w)\right\rangle=\frac{\alpha^{\prime}}{2} \delta^{i j}\{-\ln [(z-w)(\bar{z}-\bar{w})]+\ln [(1-z \bar{w})(1-\bar{z} w)]\}
$$

and the fermionic propagators (5.6). Subsequently, they can couple at the boundary to the $\lambda$-fermions via the interaction (5.7). Furthermore, since $\partial \widetilde{X}$ is holomorphic, we can use Cauchy's formula to express it in terms of the boundary data. After integrating over the interaction position $z$, we obtain the correlation functions

$$
\left\langle\partial X^{j}\left(u_{1}\right) \partial X^{k}\left(u_{2}\right)\right\rangle_{H}=\frac{i}{16 \pi} \alpha^{\prime} \oint d u \partial \widetilde{X}^{i}(u) \frac{H_{i j k}}{\left(u_{1}-u_{2}\right)^{2}} \ln \left(\frac{u_{2}-u}{u_{1}-u}\right)
$$

\footnotetext{
${ }^{7}$ These "dielectric" couplings involve also RR fluxes which are beyond the scope of our sigma model considerations.

${ }^{8}$ Since we do not discuss here the derivatives of fluxes, we will consider the case of constant $H_{i j k}$.
} 


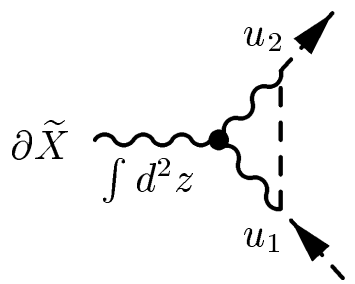

A

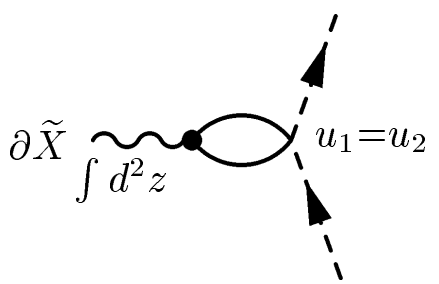

B

Figure 6: The one-loop boundary fermion-string interactions involving the correlation functions of Eqs.(6.3) $\rightarrow(\mathrm{A})$ and $(6.4) \rightarrow(\mathrm{B})$. The dotted vertices represent the H-dependent couplings generated by (6.1).

$$
\left\langle\Psi^{j}\left(u_{1}\right) \Psi^{k}\left(u_{2}\right)\right\rangle_{H}=\frac{1}{8 \pi} \oint d u \partial \widetilde{X}^{i}(u) \frac{H_{i j k}}{u_{1}-u_{2}} \ln \left(\frac{u_{2}-u}{u_{1}-u}\right),
$$

where $u_{1}$ and $u_{2}$ are located on the unit circle and the integrations extend over the whole circle. [ These correlation functions can be inserted into the Feynman diagrams as shown on Fig.6 and generate ultraviolet divergent contributions already at one loop.

The diagram of Fig.6A generates the following interaction vertex between the boundary fermions and the background:

$$
I^{(6 A)}\left(u_{i}, u_{f}\right)=-\frac{\alpha^{\prime}}{8 \pi} H_{m j k} \phi_{j k} \oint d u \partial \widetilde{X}^{m}(u) \int_{u_{i}}^{u_{f}} d u_{2} \int_{u_{2}}^{u_{f}} \frac{d u_{1}}{\left(u_{1}-u_{2}\right)^{2}} \ln \left(\frac{u_{2}-u}{u_{1}-u}\right)
$$

The $u_{1}$-integral has an ultraviolet divergence at $u_{1}=u_{2}$ which can be regulated by a short-distance cutoff $\Lambda$, so that $\left|u_{1}-u_{2}\right|>\sqrt{\Lambda} .0$ By integrating over $u_{1}$, we obtain

$$
I^{(6 A)}\left(u_{i}, u_{f}\right)=\frac{\alpha^{\prime}}{16 \pi} H_{m j k} \phi_{j k} \ln \Lambda \int_{u_{i}}^{u_{f}} d u_{2} \oint \frac{\partial \widetilde{X}^{m}(u)}{u-u_{2}} d u+\text { finite }
$$

which, after using the residue theorem, yields

$$
\Phi_{m}^{(6 A)}=\frac{i \alpha^{\prime}}{8} H_{m j k} \phi_{j k} \ln \Lambda+\text { finite. }
$$

\footnotetext{
${ }^{9}$ More precisely, the $u$-circle should be considered as a limit $r \rightarrow 1^{+}$. This will be relevant later when applying the residue theorem.

${ }^{10}$ This corresponds to $\sin ^{2}\left[\frac{\left(\tau_{1}-\tau_{2}\right)}{2}\right]>\Lambda$, as previously.
} 
A similar analysis of diagram 6B shows that it is finite. We conclude that in the presence of a NS three-form background, the one loop beta function receives an additional contribution,

$$
\beta_{m}^{H(1)}=\frac{i \alpha^{\prime}}{4} H_{m j k} \phi_{j k}
$$

the same for bosonic strings as for superstrings. The corresponding potential term whose variation ensures $\beta_{m}=0$ is given by

$$
V_{H}(\phi)=i \frac{\alpha^{\prime}}{6} H_{i j k} \operatorname{Tr}\left(\phi_{i} \phi_{j} \phi_{k}\right)
$$

In this way, the "dielectric" coupling [5] appears within the framework of sigma model in superstring theory as well in bosonic string theory.

It is quite simple to extend the above discussion to the two-loop order. The only new element is the presence of three-point vertices that couple quantum fluctuation at the boundary via the bulk interaction term (6.1). After integrating over the position $z$, we obtain

$$
\begin{aligned}
\left\langle\partial X^{i}\left(u_{1}\right) \partial X^{j}\left(u_{2}\right) \partial X^{k}\left(u_{3}\right)\right\rangle_{H} & =\frac{\left(\alpha^{\prime}\right)^{2}}{8} \frac{H_{i j k}}{\left(u_{1}-u_{2}\right)\left(u_{2}-u_{3}\right)\left(u_{3}-u_{1}\right)} \\
\left\langle\partial X^{i}\left(u_{1}\right) \Psi^{j}\left(u_{2}\right) \Psi^{k}\left(u_{3}\right)\right\rangle_{H} & =\frac{i \alpha^{\prime}}{4} \frac{H_{i j k}}{\left(u_{1}-u_{2}\right)\left(u_{1}-u_{3}\right)} .
\end{aligned}
$$

We will consider below the cases of bosonic string and superstring separately.

It is a matter of a straightforward but tedious analysis to show that in bosonic string theory the only non-vanishing contributions to the anomalous dimensions and to the beta function come from diagrams shown on Fig.7. They yield

$$
\begin{aligned}
\gamma_{H}^{(2)} & =-i\left(\alpha^{\prime}\right)^{2} H_{i j k} \phi_{i} \phi_{j} \phi_{k} \\
\beta_{m}^{H(2)} & =\frac{i}{2}\left(\alpha^{\prime}\right)^{2}\left[\phi_{i m}, H_{i j k} \phi_{j k}\right] .
\end{aligned}
$$

In order to read out the corresponding correction to the world-volume potential one has to combine Eq. (6.13) with the beta function of Eq.(4.11). It turns out that the additional 


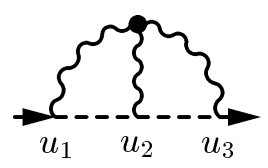

A

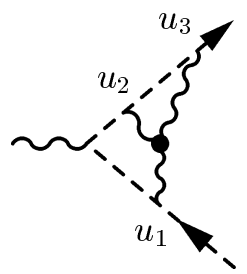

B

Figure 7: Two-loop diagrams involving the three-point vertex (6.10).

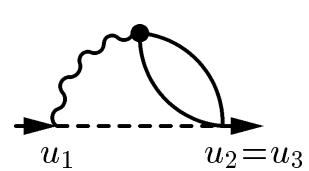

A

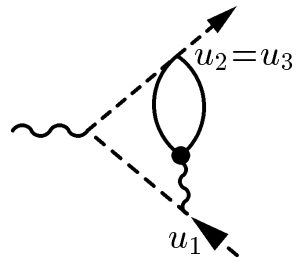

B

Figure 8: Two-loop diagrams with the fermionic vertex (6.11).

contribution combines with the second term of Eq.(4.11) to a term of the form $2 \alpha^{\prime}\left[\phi_{i m}, \beta_{i}^{(1)}\right]$, where $\beta_{i}^{(1)}$ includes now the $H$-dependent one-loop correction (6.8). Since such a term is of order $O\left[\left(\alpha^{\prime}\right)^{3}\right]$, the world-volume potential does not receive any $H$-dependent corrections at two loops.

In superstring theory there are extra contributions coming from diagrams $8 \mathrm{~A}$ and 8B, see Fig.8. In fact, they cancel against diagrams 7A and 7B, respectively. Thus in the supersymmetric case, the world-volume D-brane potential is

$$
V(\phi)=\frac{\alpha^{\prime}}{2} \operatorname{Tr}\left(\phi_{i j} \phi_{j i}\right)+i \frac{\alpha^{\prime}}{6} H_{i j k} \operatorname{Tr}\left(\phi_{i} \phi_{j} \phi_{k}\right)+O\left[\left(\alpha^{\prime}\right)^{3}\right]
$$

The absence of $O\left[\left(\alpha^{\prime}\right)^{2}\right]$ corrections agrees with Myers' proposal [5], as explained below.

The world-volume potential proposed by Myers [5] has the form巴

$$
V(\phi)=\operatorname{STr} \sqrt{\operatorname{det}\left\{\delta_{j}^{i}+2 \pi \alpha^{\prime} i \phi^{i k}\left[G_{k j}(\phi)+B_{k j}(\phi)\right]\right\}}
$$

\footnotetext{
${ }^{11}$ We set the $\mathrm{D} p$-brane tension $T_{p}=1$.
} 
where $G_{k j}(\phi)$ and $B_{k j}(\phi)$ are the transverse metric and the NS two-form, respectively, whose space-time dependence has been converted into a functional dependence on scalar matrices by means of a non-abelian Taylor expansion. In particular,

$$
B_{j k}(\phi)=\left.\exp \left[2 \pi \alpha^{\prime} \phi^{i} \partial_{X^{i}}\right] B_{j k}(X)\right|_{X^{i}=0}=B_{j k}(0)+2 \pi \alpha^{\prime} \phi^{i} \partial_{i} B_{j k}(0)+\ldots
$$

The symmetric trace STr prescription [9] applied to Eq.(6.15) amounts to formally expanding the square root of the determinant followed by taking the $U(N)$ trace symmetrized over all possible orderings of the $\phi^{i}$ and $\phi^{i j}$ matrices. Note that at the $O\left(\alpha^{\prime}\right)$ order, the potential (6.15) does correctly reproduce Eq. 6.14) modulo constant factors. At the next order, the only contribution involving one derivative of $B_{j k}$, and hence its field strength $H_{i j k}$, could arise from the $\sqrt{\text { det }}$ term $\phi_{i k} \phi_{j k}\left(\phi_{m} \partial_{m} B_{i j}\right)$. However, by applying the STr prescription, one finds that this term vanishes after symmetrizing over $\phi_{i k}$ and $\phi_{j k}$. Hence, the absence of $O\left[\left(\alpha^{\prime}\right)^{2}\right]$ corrections to D-brane potentials lends support to the STr prescription. though many authors have previously expressed their skepticism as to the validity of this prescription at higher orders, it certainly holds up to two loops in the sigma model $\alpha^{\prime}$ expansion.

\section{Acknowledgments}

We are grateful to Alexander Barabanschikov, Eric D'Hoker, Zurab Kakushadze, Boris Pioline, Stephan Stieberger and Cumrun Vafa for illuminating discussions and correspondence.

\footnotetext{
${ }^{12}$ There are two types of potential terms,$H_{i j k} \operatorname{Tr}\left(\phi_{i j} \phi_{k} \phi_{m} \phi_{m}\right)$ and $H_{i j k} \operatorname{Tr}\left(\phi_{i j} \phi_{m} \phi_{k} \phi_{m}\right)$, allowed at that level by gauge and Lorentz invariance, so this is indeed a nontrivial check.
} 


\section{References}

[1] For recent reviews, see: J.H. Schwarz, hep-th/0103165; A.A. Tseytlin, hep-th/9908105.

[2] A.A. Tseytlin, Nucl. Phys. B 276 (1986) 391 [Erratum-ibid. B 291 (1987) 876].

[3] H. Dorn and H.J. Otto, Z. Phys. C 32 (1986) 599.

[4] D. Brecher and M.J. Perry, Nucl. Phys. B 527 (1998) 121, hep-th/9801127.

[5] R.C. Myers, JHEP 9912 (1999) 022, hep-th/9910053.

[6] E. D'Hoker and D.G. Gagné, Nucl. Phys. B 467 (1996) 272, hep-th/9508131.

[7] S. Samuel, Nucl. Phys. B 149 (1979) 517;

R.A. Brandt, F. Neri, D. Zwanziger, Phys. Rev. D 19 (1979) 1153;

J.L. Gervais, A. Neveu, Nucl. Phys. B 163 (1980) 189;

I. Ya. Arefyeva, Phys. Lett. B 93 (1980) 347;

H. Dorn, Nucl. Phys. B 494 (1997) 105; JHEP 9804 (1998) 013.

[8] J. Scherk and J.H. Schwarz, Nucl. Phys. B 81 (1974) 118.

[9] A.A. Tseytlin, Nucl. Phys. B 501 (1997) 41, hep-th/9701125.

[10] See, e.g., I.R. Klebanov and L. Thorlacius, Phys. Lett. B 371 (1996) 51, hepth/9510200.

[11] M.R. Garousi and R.C. Myers, JHEP 0011 (2000) 032, hep-th/0010122;

A. Fotopoulos, "Aspects of Duality in Quantum Field Theory and Superstring Theory," Ph.D. Thesis, Northeastern University (2001). 